\title{
SPECIAL MEASURES AND REPLETENESS
}

\section{EL-BACHIR YALLAOUI}

Department of Mathematics

Polytechnic University

Six Metro Tech Center

Brooklyn, NY 11201

(Received November 14, 1989 and in revised form February 1, 1990)

\begin{abstract}
Let $X$ be an abstract set and $\mathcal{L}$ a lattice of subsets of $X$. To each lattice-regular measure $\mu$, we associate two induced measures $\hat{\mu}$ and $\dot{\mu}$ on suitable lattices of the Wallman space $I_{R}(L)$ and another measure $\mu^{\prime}$ on the space $I_{R}^{\sigma}(L)$. We will investigate the reflection of smoothness properties of $\mu$ onto $\hat{\mu}, \hat{\mu}$ and $\mu^{\prime}$; and try to set some new criterion for repleteness and measure repleteness.
\end{abstract}

KEY WORDS AND PHRASES. Replete and measure replete lattices, Lattice regular measure, Wallman space and remainder, $\sigma$-smooth, $\tau$-smooth and tight measures, purely finitely additive measures, purely $\sigma$-additive measures, purely $\tau$-additive measures.

\section{AMS SUBJECT CLASSIFICATION. $28 \mathrm{C} 15$.}

1. INTRODUCTION: Let $X$ be an abstract set and $\mathcal{L}$ a lattice subsets of $X$. To each lattice regular measure $\mu$, we associate following Bachman and Szeto [1], two induced measures $\hat{\mu}$ and $\tilde{\mu}$ on suitable lattices of subsets of the Wallman space $I_{R}(L)$ of $(X, \mathcal{L})$; we also associate to $\mu$ a measure $\mu^{\prime}$ on the space $I_{R}^{\mathrm{o}}(L)$ (see below for definitions). We give in section 2 , a brief review of the lattice notation and terminology relevant to the paper. We will be consistent with the standard terminology as used, for example, in Alexandroff [2], Frolik [3], Grassi [4], and Nöbeling [5]. We also give a brief review of the principal Theorems of [1] that we need in order to make the paper reasonably self-contained.

\section{DEFINITIONS AND NOTATIONS}

Let $X$ be an abstract set, then $L$ is a lattice of subsets of $X$ if for $A, B \subset X$ then $A \cup B \in \mathcal{L}$ and $A \cap B \in \mathcal{L}$. Throughout this work we will always assume that $\varnothing$ and $X$ are in $L$. If $A \subset X$ then we will denote the complement of $A$ by $A^{\prime}$ i.e. $A^{\prime}=X-A$. If $\mathcal{L}$ is a Lattice of subsets of $X$ then $L^{\prime}$ is defined $L^{\prime}=\left\{L^{\prime} \mid L \in L\right\}$.

\section{Lattice Terminology}

DEFINITIONS 2.1. Let $L$ be a Lattice of subsets of $X$. We say that:

1- $\quad L$ is a $\delta$-Lattice if it is closed under countable intersections.

2- $L$ is separating or $T_{1}$ if for $x, y \in X ; x \neq y$ then $\exists L \in L$ such that $x \in L$ and $y \notin L$.

3- $\quad L$ is Hausdorff or $T_{2}$ if for $x, y \in X ; x \neq y$ then $\exists A, B \in \mathcal{L}$ such that $x \in A^{\prime}, y \in B^{\prime}$ and $A^{\prime} \cap B^{\prime}=\varnothing$.

4- $\quad \mathcal{L}$ is disjunctive if for $x \in X$ and $L \in \mathcal{L}$ where $x \notin L ; \exists A, B \in \mathcal{L}$ such that $x \in A, L \subset B$ and $A \cap B=\varnothing$.

5- $\mathcal{L}$ is regular if for $x \in X, L \in \mathcal{L}$ and $x \notin L ; \exists A, B \in \mathcal{L}$ such that $x \in A^{\prime}, L \subset B^{\prime}$ and $A^{\prime} \cap B^{\prime}=\varnothing$. 
6- $\quad \mathcal{L}$ is normal if for $A, B \in \mathcal{L}$ where $A \cap B=\varnothing \exists \tilde{A}, \tilde{B} \in \mathcal{L}$ such that $A \subset \tilde{A^{\prime}, B} \subset \tilde{B}^{\prime}$ and $\tilde{A}^{\prime} \cap \tilde{B}^{\prime}=\varnothing$.

7- $\quad L$ is compact if $X=\cup_{\alpha} L_{\alpha}^{\prime}$ where $L_{\alpha} \in \mathcal{L}$ then there exists a finite number of $L_{\alpha}$ that $\operatorname{cover} X$ i.e.

$X=\bigcup_{i=1}^{n} L^{\prime}{ }_{\omega}$ where $L^{\prime}{ }_{\omega} \in \mathcal{L}$.

$A(\mathcal{L})=$ the algebra generated by $\mathcal{L}$.

$\sigma(L)=$ the $\sigma$-algebra generated by $\mathcal{L}$.

$\delta(L)=$ the Lattice of countable intersections of sets of $L$.

$\tau(L)=$ the Lattice of arbitrary intersection of sets of $L$.

$\rho(\mathcal{L})=$ the smallest class containing $\mathcal{L}$ and closed under countable unions and intersections.

If $A \in \mathcal{A}(\mathcal{L})$ then $A=\bigcup_{i=1}^{n}\left(L_{i}-\tilde{L}_{i}^{\prime}\right)$ where the union is disjoint and $L_{i}, \tilde{L}, \in \mathcal{L}$.

\section{Measure Terminology}

Let $L$ be a lattice of subsets of $X . M(L)$ will denote the set of finite valued bounded finitely additive measures on $\mathcal{A}(L)$. Clearly since any measure in $M(L)$ can be written as a difference of two non-negative measures there is no loss of generality in assuming that the measures are non-negative, and we will assume so throughout this paper.

\section{DEFINITIONS 2.2.}

1- A measure $\mu \in M(L)$ is said to be $\sigma$-smooth on $L$ if for $L_{n} \in L$ and $L_{n} \downarrow \varnothing$ then $\mu\left(L_{n}\right) \rightarrow 0$.

2- A measure $\mu \in M(L)$ is said to be $\sigma$-smooth on $\mathcal{A}(L)$ if for $A_{n} \in \mathcal{A}(L), A_{n} \downarrow \varnothing$ then $\mu\left(A_{n}\right) \rightarrow 0$.

3- $\quad$ A measure $\mu \in M(L)$ is said to be $\tau$-smooth on $L$ if for $L_{\alpha} \in L, \alpha \in \Lambda, L_{a} \downarrow \varnothing$ then $\mu\left(L_{\alpha}\right) \rightarrow 0$.

4- $\quad$ A measure $\mu \in M(L)$ is said to be $L$-regular if for any $A \in \mathcal{A}(L)$

$$
\mu(A)=\sup _{\substack{L \subset A \\ L \in C}} \mu(L)
$$

If $L$ is a lattice of subsets of $X$, then we will denote by:

$$
\begin{aligned}
& M_{R}(\mathcal{L})=\text { the set of } \mathcal{L} \text {-regular measures of } M(\mathcal{L}) \\
& M_{\sigma}(\mathcal{L})=\text { the set of } \sigma \text {-smooth measures on } \mathcal{L} \text { of } M(\mathcal{L}) \\
& M^{\mathrm{\sigma}}(L)=\text { the set of } \sigma \text {-smooth measures on } \mathcal{A}(\mathcal{L}) \text { of } M(\mathcal{L}) \\
& M_{R}^{\mathrm{o}}(\mathcal{L})=\text { the set of regular measures of } M^{\mathrm{\sigma}}(\mathcal{L}) \\
& M_{R}^{\mathrm{\tau}}(\mathcal{L})=\text { the set of } \tau \text {-smooth measures on } \mathcal{L} \text { of } M_{R}(\mathcal{L}) \\
& M_{r}^{(}(\mathcal{L})=\text { the set of tight measures on } \mathcal{L} \text { of } M_{R}(\mathcal{L}) .
\end{aligned}
$$

Clearly

$$
M_{R}^{T}(L) \subset M_{R}^{\sigma}(L) \subset M_{R}(L)
$$

DEFINITION 2.3. If $A \in \mathcal{A}(L)$ then $\mu_{x}$ is the measure concentrated at $x \in X$.

$$
\mu_{x}(A)=\left\{\begin{array}{l}
1 \text { if } x \in A \\
0 \text { if } x \notin A
\end{array}\right.
$$

$I(L)$ is the subset of $M(L)$ which consists of non-trivial zero-one measures which are finitely additive on $\mathcal{A}(L)$.

$$
\begin{aligned}
& I_{R}(\mathcal{L})=\text { the set of } \mathcal{L} \text {-regular measures of } I(\mathcal{L}) \\
& I_{\sigma}(\mathcal{L})=\text { the set of } \sigma \text {-smooth measures on } \mathcal{L} \text { of } I(\mathcal{L}) \\
& I^{\sigma}(L)=\text { the set of } \sigma \text {-smooth measures on } \mathcal{A}(\mathcal{L}) \text { of } I(\mathcal{L})
\end{aligned}
$$




$$
\begin{aligned}
& I_{\tau}(L)=\text { the set of } \tau \text {-smooth measures on } \mathcal{L} \text { of } I(L) \\
& I_{R}^{\mathrm{o}}(\mathcal{L})=\text { the set of } \mathcal{L} \text {-regular measures of } I^{\mathrm{o}}(\mathcal{L}) \\
& I_{R}^{\mathrm{\tau}}(\mathcal{L})=\text { the set of } \mathcal{L} \text {-regular measures of } I_{\mathrm{r}}(\mathcal{L})
\end{aligned}
$$

DEFINITION 2.4: If $\mu \in M(L)$ then we define the support of $\mu$ to be:

$$
S(\mu)=\bigcap\{L \in L / \mu(L)=\mu(X)\} .
$$

Consequently if $\mu \in I(L)$

$$
S(\mu)=\cap\{L \in L / \mu(L)=1\}
$$

DEFINITION 2.5. If $\mathcal{L}$ is a Lattice of subsets of $X$, we say that $L$ is replete if for any $\mu \in I_{R}^{\circ}(L)$ then $S(\mu) \neq \varnothing$.

DEFINITION 2.6. Let $L$ be a lattice of subsets of $X$. We say that $L$ is measure replete if $S(\mu) \neq \varnothing$ for all $\mu \in M_{R}^{\mathrm{o}}(L), \mu \neq 0$.

\section{Separation Terminology}

Let $L_{1}$ and $L_{2}$ be two Lattices of subsets of $X$.

DEFINITION 2.7. $L_{1}$ separates $L_{2}$ if for $A_{2}, B_{2} \in L_{2}$ and $A_{2} \cap B_{2}=\varnothing$ then there exists $A_{1}, B_{1} \in L_{1}$ such that $A_{2} \subset A_{1}, B_{2} \subset B_{1}$ and $A_{1} \cap B_{2}=\varnothing$.

REMARK 2.1. We now list few known facts found in [1] which will enable us to characterize some previously defined properties in a measure theoretic fashion.

1. $L$ is disjunctive if and only if $\mu_{x} \in I_{R}(\mathcal{L}), \forall x \in X$.

2. $L$ is regular if and only if for any $\mu_{1}, \mu_{2} \in I(\mathcal{L})$ such that $\mu_{1} \leq \mu_{2}$ on $\mathcal{L}$ we have $S\left(\mu_{1}\right)=S\left(\mu_{2}\right)$.

3. $L$ is $T_{2}$ if and only if $S(\mu)=\varnothing$ or a singleton for any $\mu \in I(L)$.

4. $L$ is compact if and only if $S(\mu) \neq \varnothing$ for any $\mu \in I_{R}(L)$.

\section{THE INDUCED MEASURES}

If $\mathcal{L}$ is a disjunctive lattice of subsets of an abstract set $X$ then there is a Wallman space associated with it. We will briefly review the fundamental properties of this Wallman space, and then associate with a regular lattice measure $\mu$, two measures $\bar{\mu}$ and $\hat{\mu}$ on certain algebras in the Wallman space (see [1]). We then investigate how properties of $\mu$ reflect to those of $\hat{\mu}$ and $\tilde{\mu}$, and conversely, then give a variety of applications of these results. Let $X$ be an abstract set and $\mathcal{L}$ a disjunctive lattice of subsets of $X$ such that $\varnothing$ and $X$ are

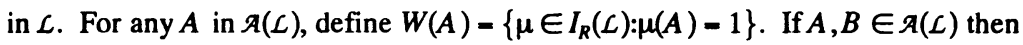

1) $\quad W(A \cup B)=W(A) \cup W(B)$.

2) $W(A \cap B)=W(A) \cap W(B)$.

3) $W\left(A^{\prime}\right)=W(A)^{\prime}$.

4) $W(A) \subset W(B)$ if and only if $A \subset B$.

5) $W(A)=W(B)$ if and only if $A=B$.

6) $W[\mathcal{A}(\mathcal{L})]=\mathcal{A}[W(\mathcal{L})]$.

Let $W(L)=\left\{W(L), L \in \mathcal{L}\right.$. Then $W(L)$ is a compact lattice of $I_{R}(L)$, and $I_{R}(L)$ with $\tau W(L)$ as the topology of closed sets is a compact $T_{1}$ space (the Wallman space) associated with the pair $X, L$. It is a $T_{2}$-space if and only if $\mathcal{L}$ is normal. For $\mu \in M(L)$ we define $\hat{\mu}$ on $\mathcal{A}(W(L))$ by: $\hat{\mu}(W(A))-\mu(A)$ where $A \in \mathcal{A}(L)$. Then $\hat{\mu} \in M(W(L))$, and $\hat{\mu} \in M_{R}(W(L))$ if and only if $\mu \in M_{R}(L)$. 
Finally, since $\tau W(L)$ and $W(L)$ are compact lattices, and $W(L)$ separates $\tau W(L)$, then $\hat{\mu}$ has a unique extension to $\tilde{\mu} \in M_{R}(\tau W(L))$. We note that by compactness $\hat{\mu}$ and $\tilde{\mu}$ are in $M_{R}^{\tau}(W(L))$ and $M_{R}^{\tau}(\tau W(L))$ respectively, where they are certainly $\tau$-smooth and of course $\sigma$-smooth. $\hat{\mu}$ can be extended to $\sigma(W(L))$ where it is $\delta W(L)$-regular; while $\tilde{\mu}$ can be extended to $\sigma(\tau(W(L)))$, the Borel sets of $I_{R}(L)$, and is $\tau W(L)$-regular on it. One is now concerned with how further properties of $\mu$ reflect over to $\hat{\mu}$ and $\bar{\mu}$ respectively. The following are known to be true (see [1]) and we list them for the reader's convenience.

THEOREM 3.1. Let $L$ be a separating and disjunctive lattice of subsets of $X$, and let $\mu \in M_{k}(\mathcal{L})$. Then

1. $\mu \in M_{R}^{\mathrm{g}}(\mathcal{L})$ if and only if $\hat{\mu}(X)=\hat{\mu}\left(I_{R}(L)\right)$.

2. $\mu \in M_{R}^{\tau}(L)$ if and only if $\tilde{\mu}(X)=\tilde{\mu}\left(I_{R}(L)\right)$.

3. If $\mathcal{L}$ is also normal (or $T_{2}$ ) then $\mu \in M_{R}^{\tau}(L)$ if and only if $X$ is $\ddot{\mu}$-measurable and $\ddot{\mu}(X)=\tilde{\mu}\left(I_{R}(L)\right.$ ).

We now give some further results related to the induced measures $\hat{\mu}$ and $\tilde{\mu}$.

THEOREM 3.2. Let $\mathcal{L}$ be a separating and disjunctive lattice, and $\mu \in M_{R}(L)$ then $\tilde{\mu}$ is $W(L)$ regular on $(\tau W(L))^{\prime}$.

PROOF. We know that $W(L)$ and $\tau W(L)$ are compact lattices and that $W(L)$ separates $\tau W(L)$. Since $\mu \in M_{R}(L)$ then $\hat{\mu} \in M_{R}[W(L)]$. Extend $\hat{\mu}$ to $\tau W(L)$. The extension is

$$
\tilde{\mu} \in M_{R}[\tau W(L)]=M_{R}^{\mathrm{o}}[\tau W(L)]=M_{R}^{\tau}[\tau W(L)]=M_{R}^{\tau}[\tau W(L)] .
$$

Let $0 \in[\tau W(L)]^{\prime}$ then since $\tilde{\mu} \in M_{R}[\tau W(L)]$ there exists $F \in \tau W(L), F \subset 0$ and

$$
|\tilde{\mu}(0)-\tilde{\mu}(F)|<\varepsilon ; \varepsilon>0 .
$$

Since $F \in \tau W(L), F=\bigcap_{\alpha \in \Lambda} W\left(L_{\alpha}\right), L_{\alpha} \in \mathcal{L}$. Also since $F \subset 0$ then $F \cap 0^{\prime}=\varnothing$ i.e. $\bigcap_{\alpha} W\left(L_{\alpha}\right) \cap 0^{\prime}=\varnothing$ by compactness there must exist $\alpha_{0} \in \wedge$ such that $W\left(L_{\mathrm{\alpha} 0}\right) \cap 0^{\prime}=\varnothing$ thus $\stackrel{\alpha}{F} \subset W\left(L_{\mathrm{\alpha} 0}\right) \subset 0^{\prime \prime}=0$ so

$$
\left|\tilde{\mu}(0)-\tilde{\mu}\left(W\left(L_{\mathrm{c} 0}\right)\right)\right|<\epsilon
$$

i.e. $\tilde{\mu}$ is $W(L)$ regular on $(\tau W(L))^{\prime}$.

THEOREM 3.3. Let $\mu \in M_{R}(L)$ then $\hat{\mu}^{*}=\bar{\mu}$ on $\tau W(L)$.

PROOF. Since $\mu \in M_{R}(L)$ and $W(L)$ is compact then $\hat{\mu} \in M_{R}[W(L)]=M_{R}^{\tau}[W(L)]$ and since $W(L)$ separates $\tau W(L)$ and $\tau W(L)$ is compact then $\tilde{\mu} \in M_{R}[\tau W(L)]=M_{R}^{\tau}[\tau W(L)]$ furthermore $\tilde{\mu}$ extends $\hat{\mu}$ to $\tau W(L)$ uniquely. Let $F \in \tau W(L)$ then

$$
\hat{\mu}(F)=\inf \sum_{i=1}^{\infty} \hat{\mu}\left(A_{i}\right), F \subset \bigcup_{i=1}^{\infty} A_{i} \text { and } A_{i} \in \mathcal{A}[W(L)]
$$

and since $\hat{\mu} \in M_{R}^{\tau}[W(L)]$ then

$$
\hat{\mu}\left(A_{\imath}\right)=\inf \hat{\mu}\left[W\left(L^{\prime},\right)\right], A_{\imath} \subset W\left(L^{\prime},\right), L_{\imath} \in \mathcal{L}
$$

thus $F \subset \bigcup_{i=1}^{\infty} W\left(L^{\prime},\right)$ but since $W(L)$ is compact then $F \subset \bigcup_{i=1}^{n} W\left(L^{\prime},\right)=W\left(L^{\prime}\right)$ where $L \in \mathcal{L}$ and

$$
\hat{\mu}^{*}(F)=\inf \hat{\mu}\left[W\left(L^{\prime}\right)\right] ; F \subset W\left(L^{\prime}\right) \text { and } L \in \mathcal{L}
$$

Now $F \subset W\left(L^{\prime}\right) \Rightarrow F \cap W(L)=\varnothing$ then since $W(L)$ separates $\tau W(L) \exists \tilde{L} \in \mathcal{L}$ such that $F \subset W(\tilde{L})$ and $W(\tilde{L}) \cap W(L)=\varnothing$. Therefore $W(\tilde{L}) \subset W\left(L^{\prime}\right)$ and hence 


$$
\hat{\mu}(F)=\inf \hat{\mu}[W(\tilde{L})]: \text { where } F \subset W(\tilde{L}) ; \tilde{L} \in \mathcal{L}
$$

i.e. that $\hat{\mu}^{\bullet}$ is regular on $\tau W(L)$. On the other hand since $\tau W(L)$ is $\delta$ then

$$
F=\bigcap_{\alpha} W\left(L_{\alpha}\right) \text { and } \bar{\mu}\left[\bigcap_{\alpha} W\left(L_{\alpha}\right)\right]=\inf _{\alpha} \bar{\mu}\left(W\left(L_{\alpha}\right)\right)=\inf \hat{\mu}\left(W\left(L_{\alpha}\right)\right)
$$

where $F \subset W\left(L_{\alpha}\right), L_{\alpha} \in \mathcal{L}$. Therefore $\hat{\mu}^{*}=\tilde{\mu}$ on $\tau W(L)$.

THEOREM 3.4. Let $L_{1}$ and $L_{2}$ be two lattices of subsets of $X$ such that $L_{1} \subset L_{2}$ and $L_{1}$ separates $L_{2}$ If $\nu \in M_{R}^{\mathrm{o}}\left(L_{2}\right)$ then $\nu=\mu^{*}$ on $L^{\prime}{ }_{2}$ and $\nu=\mu_{*}$ on $L_{2}^{\prime}$ where $\mu=\left.v\right|_{L_{1}}$.

PROOF. Let $v \in M_{R}^{\mathrm{g}}\left(L_{2}\right)$ then since $L_{1}$ separates $L_{2}, \mu \in M_{R}^{\mathrm{o}}\left(L_{1}\right)$. Since $L_{1} \subset L_{2}$ then $\sigma\left(L_{2}\right) \subset \sigma\left(L_{2}\right)$; Let $\in \subset X$ then

$$
v^{*}(E)=\inf _{E \subset B, B \in \mathcal{\sigma}\left(L_{2}\right)} v(B) \leq \inf _{E \subset A, A \in \sigma\left(L_{1}\right)} v(A)=\mu^{*}(E)
$$

therefore, $v^{*} \leq \mu^{*}$. Now on $L_{2}, v^{*}=\leq \mu^{*}$. Suppose $\exists L_{2} \in L_{2}$ such that $v\left(L_{2}\right)<\mu^{*}\left(L_{2}\right)$ then since

$$
v \in M_{R}^{\mathrm{o}}\left(L_{2}\right), v\left(L_{2}\right)=\inf v\left(\tilde{L}^{\prime}{ }_{2}\right), L_{2} \subset \tilde{L}^{\prime}{ }_{2} \text { and } \tilde{L}_{2} \in L_{2}
$$

then $L_{2} \cap \tilde{L}_{2}=\varnothing$ and by separation $\exists L_{1}, \tilde{L}_{2} \in \mathcal{L}_{1}$ such that $L_{2} \subset L_{1}, \subset \tilde{L}_{1}^{\prime} \subset \tilde{L}_{2}^{\prime}$ and therefore

$$
\begin{aligned}
v\left(L_{2}\right) & =\inf _{\alpha} \mu\left(L_{1 \alpha}\right) \text { where } L_{2} \subset L_{1 \alpha} \\
& =\inf _{\beta} v\left(\tilde{L}_{2 \beta}^{\prime}\right) \text { where } L_{2} \subset \tilde{L}_{2 \beta}^{\prime} \\
& <\mu^{\prime}\left(L_{2}\right)
\end{aligned}
$$

$\forall \varepsilon>0 \exists L_{1} \in L_{1}$ such that $L_{2} \subset L_{1}$ and $\mu\left(L_{1}\right)-\varepsilon<v\left(L_{2}\right)<\mu\left(L_{1}\right)$ but since $L_{2} \subset L_{1}$ then $\mu^{*}\left(L_{2}\right) \leq \mu\left(L_{1}\right)<$ $v\left(L_{2}\right)+\varepsilon$ which is a contradiction to our assumption. Therefore $v=\mu^{*}$ on $L_{2}$ and thus $v=\mu$. on $L^{\prime}{ }_{2}$ This theorem is a generalization of the previous one in which we used the compactness of $W(L)$ to have a regular restriction of the measure. Next consider the space $I_{R}^{\sigma}(L)$ and the induced measure $\mu^{\prime}$.

DEFINITION 3.1. Let $\mathcal{L}$ be a disjunctive lattice of subsets of $X$.

1) $\quad W_{\mathrm{\sigma}}(L)=\left\{\mu \in I_{R}^{\mathrm{o}}(L) \mid \mu(L)=1\right\} ; L \in \mathcal{L}$

2) $W_{\mathrm{\sigma}}(L)=\left\{W_{\mathrm{\sigma}}(L), L \in L\right\}$

3) $W_{\sigma}(A)=\left\{\mu \in I_{R}^{\mathrm{o}}(L) \mid \mu(A)=1\right\}, A \in \mathcal{A}(L)$

4) $W_{\mathrm{o}}(\mathcal{L})=W(L) \cap I_{R}^{\mathrm{o}}(\mathcal{L})$

The following properties hold:

PROPOSITION 3.1. Let $L$ be a disjunctive lattice then for $A, B \in \mathcal{A}(L)$

1) $\quad W_{\mathrm{o}}(A \cap B)=W_{\mathrm{\sigma}}(A) \cup W_{\mathrm{o}}(B)$

2) $\quad W_{\mathrm{o}}(A \cup B)=W_{\mathrm{o}}(A) \cap W_{\mathrm{o}}(B)$

3) $W_{\mathrm{o}}\left(A^{\prime}\right)=W_{\mathrm{o}}(A)^{\prime}$

4) $W_{\mathrm{o}}(A) \subset W_{\mathrm{o}}(B)$ if and only if $A \subset B$

5) $\quad \mathcal{A}\left[W_{\mathrm{o}}(L)\right]=W_{\mathrm{o}}[\mathcal{A}(L)]$

The proof is the same as for $W(L)$ by simply using the properties of $W(L)$ and the fact that $W_{\mathrm{\sigma}}(A)=W(A) \cap I_{R}^{\mathrm{\sigma}}(L)$ and $W_{\mathrm{o}}(B)=W(B) \cap I_{R}^{\mathrm{o}}(L)$.

REMARK 3.1. It is not difficult to show that $\sigma\left[W_{\sigma}(L)\right]=W_{\sigma}[\sigma(L)]$. Also, for each $\mu \in M(L)$ we define $\mu^{\prime}$ on $\mathcal{A}\left[W^{\prime}{ }_{\mathrm{o}}(\mathcal{L})\right]$ as follows: 


$$
\mu^{\prime}\left[W_{\mathrm{o}}(A)\right]=\mu(A) \text { where } A \in \mathcal{A}(L)
$$

$\mu^{\prime}$ is defined and the map $\mu \rightarrow \mu^{\prime}$ from $M(L)$ to $M\left(W_{\sigma}(L)\right)$ is onto. In addition, it can readily be checked that,

THEOREM 3.5. Let $L$ be disjunctive then
1) $\mu \in M(L)$ if and only if $\mu^{\prime} \in M\left[W_{\sigma}(L)\right]$
2) $\mu \in M_{R}(L)$ if and only if $\mu^{\prime} \in M_{R}\left[W_{\mathrm{o}}(L)\right]$
3) $\mu \in M_{\mathrm{o}}(L)$ if and only if $\mu^{\prime} \in M_{\sigma}\left[W_{\mathrm{\sigma}}(L)\right]$
4) $\mu \in M^{\sigma}(L)$ if and only if $\mu^{\prime} \in M^{\sigma}\left[W_{\sigma}(L)\right]$
5) $\quad \mu \in M_{R}^{\sigma}(L)$ if and only if $\mu^{\prime} \in M_{R}^{\sigma}\left[W_{\mathrm{o}}(L)\right]$

THEOREM 3.6. Let $\mathcal{L}$ be a separating and disjunctive lattice of subsets of $X$, and let $\mu \in M_{R}^{\mathrm{o}}(\mathcal{L})$. Then

1. $\quad \mu^{\prime} \in M_{R}^{\tau}\left(W_{\mathrm{\sigma}}(L)\right)$ if and only if $\hat{\mu}^{*}\left(I_{R}^{\mathrm{o}}(L)\right)=\hat{\mu}\left(I_{R}(L)\right)$.

2. If $L$ is also normal or $T_{2}$ then $\mu^{\prime} \in M_{R}^{\tau}\left(W_{\mathrm{o}}(L)\right)$ if and only if $I_{R}^{\mathrm{o}}(L)$ is $\tilde{\mu}^{*}$-measurable and $\ddot{\mu}\left(I_{R}^{\mathrm{o}}(L)\right)=\tilde{\mu}\left(I_{R}(L)\right)$.

We note some consequences.

COROLLARY 3.1. If $L$ is a separating, disjunctive and replete lattice of subsets of $X$, then $\mu^{\prime} \in\left[M_{R}^{\mathcal{\tau}}(L)\right]$ implies $\mu \in M_{R}^{\mathcal{\tau}}(L)$.

PROOF. Since $L$ is replete then $X=I_{R}^{\mathrm{O}}(L)$ then from the previous theorem we have

$$
\tilde{\mu}\left(I_{R}(L)\right)=\ddot{\mu}\left(I_{R}^{\mathrm{\sigma}}(L)\right)=\ddot{\mu}(X)
$$

i.e. $\mu \in M_{R}^{\mathcal{T}}(\mathcal{L})$ from theorem 3.1 .

COROLLARY 3.2. Let $\mathcal{L}$ be separating and disjunctive. If $\mu^{\prime} \in M_{R}^{\tau}\left(W_{\mathrm{o}}(L)\right) \Rightarrow \mu \in M_{R}^{\tau}(L)$ then $L$ is replete.

PROOF. Let $\mu \in I_{R}^{\mathrm{\sigma}}(\mathcal{L})$ then since $W_{\mathrm{o}}(\mathcal{L})$ is replete $\mu^{\prime} \in I_{R}^{\tau}\left[W_{\mathrm{o}}(\mathcal{L})\right]$ then by hypothesis $\mu \in I_{R}^{\tau}(L)$ therefore $I_{R}^{\mathrm{o}}(\mathcal{L})=I_{R}^{\mathrm{\tau}}(\mathcal{L})$ or $\mathcal{L}$ is replete. If we combine the two corollaries we get the following:

THEOREM 3.7. Let $\mathcal{L}$ be separating and disjunctive. Then $\mathcal{L}^{\prime}$ is replete if and only if $\mu^{\prime} \in M_{R}^{\top}\left(W_{\sigma}(L)\right) \Rightarrow \mu \in M_{R}^{\tau}(L)$.

THEOREM 3.8. Let $\mathcal{L}$ be a separating, disjunctive, normal and replete lattice. Then

$$
\mu^{\prime} \in M_{R}^{\tau}\left[W_{\sigma}(L)\right] \text { if and only if } \mu \in M_{R}^{\tau}(L) .
$$

PROOF.

1. Let $\mu^{\prime} \in M_{R}^{\tau}\left[W_{\sigma}(L)\right]$ then since $\mathcal{L}$ is rep!ete then $X=I_{R}^{\mathrm{\sigma}}(\mathcal{L})$ and $X$ is $\ddot{\mu}^{*}$-measurable and

$$
\ddot{\mu}\left(I_{R}^{\mathrm{o}}(L)\right)=\tilde{\mu}\left(I_{R}(L)\right)=\tilde{\mu}(X)
$$

then by theorem 3.1 we get that $\mu \in M_{R}^{\tau}(L)$.

2. Conversely suppose $\mu \in M_{R}^{\mathcal{\tau}}(\mathcal{L})$ then from theorem 3.1 we get that

$$
\tilde{\mu}(X)=\tilde{\mu}\left(I_{R}(L)\right)
$$


and $X$ is $\ddot{\mu}$-measurable but $X \subset I_{R}^{\mathrm{\sigma}}(\mathcal{L}) \subset I_{R}(L)$ therefore $\tilde{\mu}^{*}\left(I_{R}^{\mathrm{o}}(L)\right)=\tilde{\mu}\left(I_{R}(L)\right)$, then since $\mathcal{L}$ is replete $X=I_{R}^{\mathrm{o}}(L)$ so $\tilde{\mu}^{*}(X)=\tilde{\mu}\left(I_{R}^{\mathrm{o}}(L)\right)=\tilde{\mu}\left(I_{R}(L)\right)$ then from theorems 3.1 and $3.7 \mu^{\prime} \in M_{R}^{\mathrm{q}}\left[W_{\mathrm{o}}(L)\right]$.

\section{SPECIAL MEASURES AND REPLETENESS}

In this section we define a purely finitely additive measure (p. f. a.), a purely $\sigma$-additive measure (p. $\sigma$. a.) and a purely $\tau$-additive measure (p. $\tau$. a.) and for each type we give a characterization theorem. Then we will define strong $\sigma$-additive measures (s. $\sigma$. a.) and (s. $\tau$. a.) measures and give for each a characterization theorem. Finally we will investigate relationships among these measures under repleteness.

LEMMA 4.1. Let $\mathcal{L}$ be a lattice of subsets of $X$ and $\mu \in M_{R}(L)$.

1. Consider $\hat{\mu}$ on $\sigma[W(L)]$; we saw in earlier work that $\hat{\mu}$ is $\delta(W(L))$ regular on $\sigma[W(L)]$.

Let $H \subset I_{R}(L)$ such that $\hat{\mu}^{*}(H)=a \neq 0$ then $\exists \rho$ countably additive on $\sigma[\tau W(L)]$ and $\tau-W(L)$ regular such that $0 \leq \rho \leq \hat{\mu}$ and $\rho^{\circ}(H)=\rho\left(I_{R}(L)\right)=a \neq 0$.

2. Consider $\tilde{\mu}$ in $\sigma[\tau W(L)]$; we say that $\tilde{\mu}$ is $\tau W(L)$ regular on o[ $\tau W(L)]$.

Let $H \subset I_{R}(L)$ such that $\ddot{\mu}(H)=a \neq 0$ then $\exists \rho$ countably additive on $\tau W(L)$ regular on $\sigma[\tau W(L)]$ such that $0 \leq \rho \leq \tilde{\mu}$ and $\rho^{*}(H)=\rho\left(I_{R}^{\mathrm{o}}(L)\right)=a$

\section{DEFINITION 4.1.}

1. Let $\mu \in M_{R}(L)$; we say that $\mu$ is p. f. a. if for $\gamma \in M_{\mathrm{o}}(L)$ and $0 \leq \gamma \leq \mu$ on $\mathcal{A}(L)$ then $\gamma=0$.

2. Let $\mu \in M_{R}(L)$; we say that $\mu$ is p. $\sigma$. a. if for $\gamma \in M_{\sigma}(L), \gamma \quad \tau$-smooth on $\mathcal{L}$ and $0 \leq \gamma \leq \mu$ then $\gamma=0$.

THEOREM 4.1. Let $L$ be a separating and disjunctive lattice and $\mu \in M_{R}(L)$ then:

1. $\quad \mu$ is p. f. a. $\Rightarrow \hat{\mu}^{*}(X)=0$.

2. $\quad \mu$ is p. o. a. $\Rightarrow \ddot{\mu}(X)=0$.

If we further assume that $L$ is $\delta$ and $\sigma(L)=\rho(L)$ then the converses are true.

$P R O O F$. The proof will be given only for part (1) and is similar for the second one.

1. Suppose $\mu$ is purely finitely additive. If $\hat{\mu}^{*}(X)=a \neq 0$ then from previous Lemma 4.1 there exists $\rho \in M_{R}[W(L)]=M_{R}^{\tau}[W(L)]$ such that

$$
\begin{gathered}
0 \leq \rho \leq \hat{\mu} \text { and } \rho^{*}(X)=\rho\left(I_{R}(L)\right)=a \text {; then } \\
\quad \rho=\gamma \text { and } \gamma \in M_{R}^{\sigma}(L) \text { so } \\
0 \leq \rho=\hat{\gamma} \leq \hat{\mu} \Rightarrow 0 \leq \gamma \leq \mu \Rightarrow \gamma=0
\end{gathered}
$$

from the definition of purely finitely additive which is a contradiction because

$$
\hat{\gamma}\left[I_{R}(L)\right]=a \neq 0 \text { and therefore } \hat{\mu}^{*}(X)=0 .
$$

2. Conversely if $\hat{\mu}^{*}(X)=0$ and $0 \leq \gamma \leq \mu$ on $\mathcal{A}(L)$ where $\gamma \in M_{\mathrm{o}}(L)$ and $\mathcal{L}$ is $\delta$ and $\rho(L)=\sigma(L)$ then $\gamma \in M_{R}^{\mathrm{o}}(L)$ and $0 \leq \hat{\gamma} \leq \hat{\mu}$ on $\mathcal{A}[W(L)]$ then $0 \leq \gamma \leq \hat{\mu}$ on $\mathcal{A}(L)$; and therefore

$$
0 \leq \gamma^{*} \leq \mu^{*} \text { and since } \hat{\mu}^{*}(X)=0 \Rightarrow \hat{\gamma}^{*}(X)=0=\hat{\gamma}\left[I_{R}(L)\right]
$$

hence $\gamma=0$ i.e. $\mu$ is purely finitely additive.

DEFINITIONS 4.2. Let $L$ be any lattice of subsets of $X$. 
1. Let $\mu \in M_{R}(\mathcal{L})$, we say that $\mu$ is $\sigma$. f. a. if for $\gamma$ such that $0 \leq \gamma \leq \mu$ on $\mathcal{A}(\mathcal{L})$ and $\gamma^{\prime} \in M^{\sigma}\left[W_{\sigma}(\mathcal{L})\right]$ then $\gamma=0$.

2. Let $\mu \in M_{R}^{\mathrm{g}}(\mathcal{L})$, we say that $\mu$ is s. $\sigma$. a. if for $\gamma$ such that $0 \leq \gamma \leq \mu$ on $\lambda(L)$ and $\gamma^{\prime} \in M^{\circ}\left[W_{\mathrm{o}}(L)\right], \gamma^{\prime} \tau$-smooth on $W_{\mathrm{\sigma}}(\mathcal{L})$ then $\gamma=0$.

LEMMA 4.2. Let $L$ be a disjunctive lattice of subsets of $X$. If $\lambda \in M_{R}(\tau W(L))=M_{R}^{\chi}(\tau W(L))$ and $\lambda^{*}\left(I_{R}^{\mathrm{o}}(L)\right)=\lambda\left(I_{R}(L)\right)$ then $\exists \mu \in M_{R}(L)$ such that $\lambda=\tilde{\mu}$ and $\mu^{\prime} \in M_{R}^{\mathrm{T}}\left[W_{\mathrm{o}}(L)\right]$. The proof is not difficult.

THEOREM 4.2. Let $L$ be a disjunctive lattice of subsets of $X$. Let $\mu \in M_{R}^{\mathrm{o}}(L)$ then:

1. If $\mu$ is s. $\sigma$. a. then $\ddot{\mu}\left(I_{R}^{\mathrm{\sigma}}(L)\right)=0$.

2. If $W_{\mathrm{o}}(L)$ is $\delta, \sigma\left[W_{\mathrm{o}}(L)\right]=\rho\left[W_{\mathrm{o}}(L)\right]$ and $\tilde{\mu}^{*}\left(I_{R}^{\mathrm{o}}(L)\right)=0$ then $\mu$ is s. o. a.

PROOF.

1. Suppose $\mu$ is strong $\sigma$ additive but $\ddot{\mu}^{*}\left(I_{R}^{\sigma}(L)\right)=a \neq 0$ then from lemma (4.1) $\exists \rho$ countably additive on $\sigma[\tau W(L)]$ and $\tau W(L)$ regular such that $0 \leq \rho \leq \tilde{\mu}$ and $\rho^{*}\left(I_{R}^{\sigma}(L)\right)=\rho\left(I_{R}(L)\right)=a$ from previous lemma $4.2 \rho=\bar{\gamma}$ where $\gamma^{\prime} \in M_{R}^{\tau}\left(W_{\mathrm{o}}(L)\right)$ then

$$
0 \leq \rho=\tilde{\gamma} \leq \tilde{\mu} \Rightarrow 0 \leq \bar{\gamma} \leq \tilde{\mu} \Rightarrow 0 \leq \gamma \leq \mu
$$

and since $\mu$ is s. $\sigma$. a. then $\gamma=0$ which is a contradiction to the fact that

$$
\rho\left(I_{R}(L)\right)=\tilde{\gamma}\left(I_{R}(L)\right)=a \neq 0
$$

and hence $\bar{\mu}^{*}\left(I_{R}^{\mathrm{\sigma}}(\mathcal{L})\right)=0$.

2. Suppose $W_{\mathrm{\sigma}}(L)$ is $\delta, \quad \sigma\left[W_{\sigma}(L)\right]=\rho\left[W_{\sigma}(L)\right]$ and $\tilde{\mu}^{*}\left(I_{R}(L)\right)=0$. Let $\gamma \in M(L), 0 \leq \gamma \leq \mu$ and $\gamma^{\prime} \in M^{\sigma}\left[W_{\mathrm{o}}(L)\right]$ and $\tau$-smooth on $W_{\mathrm{o}}(\mathcal{L})$ then $\gamma^{\prime} \in M_{R}^{\mathrm{g}}\left[W_{\mathrm{\sigma}}(L)\right]$ and even $\gamma^{\prime} \in M_{R}^{\tau}\left[W_{\mathrm{\sigma}}(\mathcal{L})\right]$. So

$$
0 \leq \tilde{\gamma} \leq \bar{\mu} \text { on } \mathcal{A}[W(L)]
$$

and therefore $0 \leq \gamma^{\prime} \leq \mu^{\prime}$ on $\mathcal{A}\left[W_{\mathrm{\sigma}}(L)\right]$. Furthermore $0 \leq \ddot{\gamma} \leq \tilde{\mu}^{*}$ and since $\tilde{\mu}^{*}\left(I_{R}^{\mathrm{o}}(L)\right)=0$ then $\tilde{\gamma}^{*}\left(I_{R}^{\mathrm{o}}(\mathcal{L})\right)=$ $\bar{\gamma}\left(I_{R}^{\sigma}(L)\right)=0$ i.e. $\gamma=0$ i.e. $\mu$ is s. $\sigma$. a.

NOTE. If $L$ is $\delta$ and $\sigma(L)=\rho(L)$ then $W_{\sigma}(L)$ is $\delta$ and $\sigma\left[W_{\sigma}(L)\right]=\rho\left(W_{\sigma}(L)\right)$ will hold.

PROPOSITION 4.1. Let $L$ be separating and disjunctive if $L$ is also $\delta$ and $\sigma(L)=\rho(L)$ then $\mu$ is s. o. a. $\Rightarrow \mu$ is p. $\sigma$. a.

PROOF. $\mu$ is s. $\sigma$. a. $\Rightarrow \ddot{\mu}\left(I_{R}^{\alpha}(L)\right)=0 \Rightarrow \ddot{\mu}^{*}(X)=0: \tilde{\mu}^{*}(X)=0$ and $L$ is $\delta$ and $\rho(L)=\sigma(L) \Rightarrow \mu$ is p. $\sigma$. a.

PROPOSITION 4.2. If $\mathcal{L}$ is disjunctive then $\mu$ is s. f. a. if and only if $\mu$ is p. f. a. PROOF.

1. Suppose $\mu$ is s. f. a. and $\gamma^{\prime} \in M_{\sigma}(L) ; 0 \leq \gamma \leq \mu$ then $\gamma^{\prime} \in M^{\sigma}\left[W_{\sigma}(L)\right]$ and $0 \leq \gamma \leq \mu \Rightarrow \gamma=0$ by s. f. a. Therefore $\mu$ is p. f. a.

2. Suppose $\mu$ is p. f. a. and $\gamma^{\prime} \in M^{\sigma}\left[W_{\mathrm{o}}(L)\right] ; 0 \leq \gamma \leq \mu$ then $\gamma \in M^{\mathrm{o}}(L)$ and $0 \leq \gamma \leq \mu \Rightarrow \gamma=0$ by purely finitely additive. Therefore $\mu$ is s. f. a.

PROPOSITION 4.3. If $\mathcal{L}$ is replete then $\mu$ is s. $\sigma$. a. if and only if $\mu$ is p. $\sigma$. a. 
PROOF. $L$ replete $\Rightarrow X=I_{R}^{\tau}(L)=I_{r}^{\mathrm{O}}(\mathcal{L})$ then $L=W_{\mathrm{\sigma}}(L)$ and so $\gamma \in M^{\mathrm{\sigma}}(L)$ and $\tau$-smooth on $\mathcal{L} \Leftrightarrow \gamma^{\prime} \in M^{\circ}\left(W_{\mathrm{o}}(\mathcal{L})\right)$ and $\tau$-smooth or $W_{\mathrm{o}}$ therefore the definitions are equivalent.

THEOREM 4.3. Suppose $L$ is separating, disjunctive and $\delta$ and $\sigma(L)=\rho(L)$ then $L$ is replete if and only if for any $\mu \in M_{R}^{\mathrm{o}}(\mathcal{L}), \mu$ is p. $\sigma$. a. $\Rightarrow \mu$ is s. $\sigma$. a.

PROOF.

1. We saw in proposition 4.3 that if $\mathcal{L}$ is replete then p. $\sigma$. a. $\Leftrightarrow$ s. $\sigma$. a.

2. Conversely suppose that $\mu$ is p. $\sigma$. a. $\Rightarrow \mu$ is s. o. a. for any $\mu \in M_{R}^{\mathrm{o}}(L)$ but $X \approx I_{R}^{\mathrm{o}}(L)$. Let $\mu \in I_{R}^{\mathrm{o}}(L)$ then $\tilde{\mu}$ is $\tau W(L)$ regular and $S(\bar{\mu})=\{\mu\}, \ddot{\mu}(X)=0$. Now since $\ddot{\mu}(X)=0, L$ is $\delta$ and $\sigma(L)=\rho(L)$ then from theorem $4.1 \mu$ is purely $\sigma$ additive by assumption; but $\mu$ is s. $\sigma$. a. $\Rightarrow \ddot{\mu}\left(I_{R}^{\mathrm{o}}(L)\right)=0$ from proposition 4.2; which is a contradiction because $\mu \in M_{R}^{\mathrm{o}}()$ and $\bar{\mu}[\{\mu\}]=1$. Therefore $X=I_{R}^{\mathrm{o}}(L)$.

DEFINITION 4.3. Let $\mu \in M_{R}^{\tau}(L)$.

1. We say that $\mu$ is p. $\tau$ a. if for $\gamma \in M_{\mathrm{o}}(\mathcal{L}), 0 \leq \gamma \leq \mu$, and $\gamma \mathcal{L}$-tight then $\gamma=0$.

2. We say that $\mu$ is s. $\tau$. a. if for $\gamma^{\prime} \in M^{\sigma}\left[W_{\mathrm{o}}(L)\right], 0 \leq \gamma \leq \mu$ on $\mathcal{A}(\mathcal{L})$ and $\gamma^{\prime}$ is $W_{\mathrm{o}}(L)$-tight then $\gamma=0$.

THEOREM 4.4. Let $L$ be a separating, disjunctive and normal lattice. If $\mu \in M_{R}^{\tau}(\mathcal{L})$ then:

1. $\quad \mu$ is p. $\tau$. a. $\Rightarrow \tilde{\mu}^{*}\left(I_{R}(L)-X\right)=\tilde{\mu}\left(I_{R}(L)\right)$.

2. $\quad \mu$ is s. $\tau$. a. $\Rightarrow \ddot{\mu}\left(I_{R}(L)-I_{R}^{\mathrm{o}}(L)\right)=\bar{\mu}\left(I_{R}(L)\right)$.

If we further assume that $\mathcal{L}$ is $\delta$ and $\sigma(L)=\rho(L)$ then the converses are true.

$P R O O F$. We will prove only the second proposition and the proof of the first is similar.

2.a) Suppose $\mu$ is s. $\tau$. a. but $\tilde{\mu}^{*}\left(I_{R}(L)-I_{R}^{\mathrm{o}}(L)\right)<\tilde{\mu}\left(I_{R}(L)\right)$, then there exists $G \in[\tau W(L)]^{\prime}$ such that $I_{R}(L)-I_{R}^{\mathrm{o}}(\mathcal{L}) \subset G$ and $\tilde{\mu}(G)<\tilde{\mu}\left(I_{R}(L)\right)$. Let $F=I_{R}(L)-G, F \in \tau W(L)$ then $F \subset I_{R}^{\mathrm{o}}(L)$ and $F$ is $W_{\mathrm{s}}(L)$ compact, for if $F \subset \cup_{\alpha} W_{\mathrm{o}}\left(L_{\alpha}\right)^{\prime} \Rightarrow F \subset \cup_{\alpha} W\left(L_{\alpha}\right)$. Therefore

$$
F \subset \underset{\text { fin }}{\cup} W\left(L_{\mathrm{\alpha} u}\right)=W(\hat{L})^{\prime} \hat{L} \in \mathcal{L}
$$

thus $F \subset W_{\mathrm{o}}(\hat{L})^{\prime}$ since $F \subset I_{R}^{\mathrm{o}}(L)$ and $\tilde{\mu}(F)>0$ since $\tilde{\mu}(G)<\tilde{\mu}\left(I_{R}(L)\right)$. Also since $W_{\mathrm{o}}(L)$ is normal and $T_{2}$ then $F \in \tau W_{\sigma}(L)$. Now $\mu \in M_{R}^{\tau}(\mathcal{L})$ projects onto $I_{R}^{\mathrm{o}}(\mathcal{L})$ and $\mu^{\prime}$ is the projection on $W_{\mathrm{o}}(\mathcal{L})$ and $\mu^{\prime \prime}$ is the projection on $\tau W_{\mathrm{o}}(L)$. For $E \in \mathcal{A}\left(W_{\mathrm{o}}(L)\right) \operatorname{let} \lambda(E)=\mu^{\prime \prime}(E \cap F)$ then $0 \leq \lambda(E) \leq \mu^{\prime \prime}(E)=\mu^{\prime}(E)$ so $0 \leq \lambda \leq \mu^{\prime}$ on $A\left[W_{\mathrm{o}}(L)\right]$. Now if

$$
W_{\sigma}\left(L_{\alpha}\right) \downarrow \varnothing, L_{\alpha} \in \mathcal{L} \text { then } W_{\sigma}\left(L_{\alpha}\right) \cap F \downarrow \varnothing \text { and } \lambda\left[W_{\sigma}\left(L_{\alpha}\right)\right]=\mu^{\prime \prime}\left[W_{\sigma}\left(L_{\alpha}\right) \cap F\right] \rightarrow 0
$$

then

$$
\lambda \in M_{R}^{\mathcal{\tau}}\left(W_{\mathrm{o}}(L)\right)
$$

Since $\lambda$ is $\tau$-smooth and $W_{\sigma}(L)$ is regular. Also $\lambda \in M_{R}^{\tau}\left[W_{\sigma}(L)\right]$ since $\forall \in>0, \lambda\left(I_{R}^{\mathrm{o}}(L)\right)=\mu^{\prime \prime}(F)$ then

$$
\begin{aligned}
\lambda^{*}(F) & =\lambda^{*}\left(\underset{\alpha}{\cap} W_{\sigma}\left(L_{\alpha}\right)\right)=\inf \lambda\left[W_{\sigma}\left(L_{\alpha}\right)\right] \\
& =\inf \mu^{\prime \prime}\left[W_{\sigma}\left(L_{\alpha}\right) \cap F\right]=\mu^{\prime \prime}\left(W_{\alpha}\left(L_{\alpha}\right) \cap F\right)=\mu^{\prime \prime}(F) .
\end{aligned}
$$

Therefore

$$
\lambda^{*}(F)=\mu^{\prime \prime}(F)=\lambda\left(I_{R}^{\mathrm{o}}(L)\right)>\lambda\left(I_{R}^{\mathrm{o}}(\mathcal{L})\right)-\varepsilon
$$


Thus

$$
\lambda \in M_{R}^{\tau}\left[W_{\mathrm{o}}(\mathcal{L})\right]
$$

Therefore

$$
\lambda=\gamma^{\prime} \in M_{R}^{\tau}\left[W_{\mathrm{o}}(\mathcal{L})\right]
$$

so

$$
0 \leq \gamma^{\prime} \leq \mu^{\prime} \text { on } A\left[W_{\sigma}(L)\right] \text { and } 0 \leq \gamma \leq \mu \text { on } \mathcal{A}(L)
$$

and $\gamma^{\prime} \in M_{R}^{q}\left[W_{\mathrm{o}}(L)\right]$ and $\lambda=\gamma^{\prime} \neq 0$ contradiction. Hence

$$
\ddot{\mu}\left(I_{R}(L)-I_{R}^{\mathrm{O}}(L)\right)=\tilde{\mu}\left(I_{R}(L)\right)
$$

2.b) Let $\gamma^{\prime} \in M_{R}^{\mathrm{o}}\left(W_{\mathrm{o}}(L)\right)$ then $\gamma \in M_{R}^{\mathrm{g}}(L)$ also $\gamma^{\prime} \in M_{R}^{\mathrm{q}}\left[W_{\mathrm{\sigma}}(L)\right]$ because $\gamma^{\prime}$ is $W_{\mathrm{o}}(L)$-tight. Now

$$
0 \leq \gamma^{\prime} \leq \mu^{\prime} \text { on } \mathcal{A}\left[W_{\mathrm{o}}(L)\right] \Rightarrow 0 \leq \gamma^{\prime \prime} \leq \mu^{\prime \prime} \text { on } \mathcal{A}\left[\tau W_{\mathrm{o}}(L)\right]
$$

also $I_{R}^{\mathrm{o}}(L)$ is $\tilde{\gamma}^{*}$-measurable since $\gamma^{\prime} \in M_{R}^{\mathrm{T}}\left[W_{\mathrm{o}}(L)\right]$ then $\tilde{\gamma}^{*}\left(I_{R}^{\mathrm{o}}(L)\right)=\tilde{\gamma}\left(I_{R}(L)\right)$ from previous work. Therefore $\exists F, W_{\mathrm{o}}(L)$-compact, $F \subset I_{R}^{\mathrm{o}}(L)$ such that

$$
\gamma^{\prime \prime}(F)>\frac{1}{2} \gamma^{\prime \prime}\left(I_{R}^{\mathrm{o}}(L)\right)=\frac{1}{2} \gamma^{\prime}\left[I_{R}^{\mathrm{o}}(L)\right]
$$

so

$$
\gamma^{\prime \prime}(F) \leq \mu^{\prime \prime}(F)=0 \text { since } F \subset I_{R}^{\mathrm{o}}(\mathcal{L})
$$

and since by hypothesis

$$
\ddot{\mu}\left(I_{R}(L)-I_{R}^{\mathrm{O}}(L)\right)=\bar{\mu}\left[I_{R}(L)\right]
$$

then

$$
\ddot{\mu}^{*}\left(I_{R}^{\mathrm{o}}(L)\right)=0
$$

and

$$
\tilde{\mu}(F)=0
$$

but then

$$
\gamma^{\prime}\left(I_{r}^{\mathrm{O}}(L)\right)=0 \Rightarrow \gamma^{\prime}=0 \Rightarrow \gamma=0
$$

therefore $\mu$ is $\mathbf{s .} \boldsymbol{\tau}$. a.

\section{REFERENCES}

1. Bachman, G. and Szeto, M., On Strongly Measure Replete Lattices, Support of a Measure and the Wallman Remainder, Per. Math. Hung, 15(2) (1981), 127-155.

2. Alexandroff, A. D., Additive Set Functions in Abstract Spaces, Mat. Sb, (N.S.) 8, 50 (1940), 307-348.

3. Frolik, Z., Prime Filter with C.I.P., Comment. Math. Univ. Carolina, 13, No. 3 (1972), 553-573.

4. Grassi, P., On Subspaces of Replete and Measure Replete Spaces, Canad. Math. Bull, 27(1), (1984), 58-64.

5. Nöbeling, G., Grundlagender Analytischen Topologie, Springer-Verlag, Berlin, 1954. 


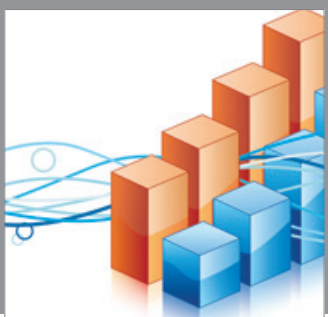

Advances in

Operations Research

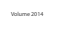

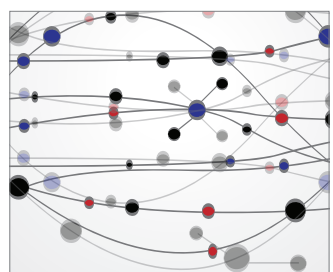

\section{The Scientific} World Journal
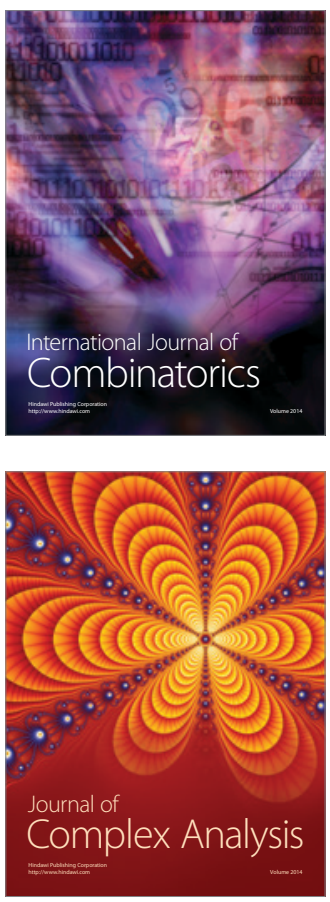

International Journal of

Mathematics and

Mathematical

Sciences
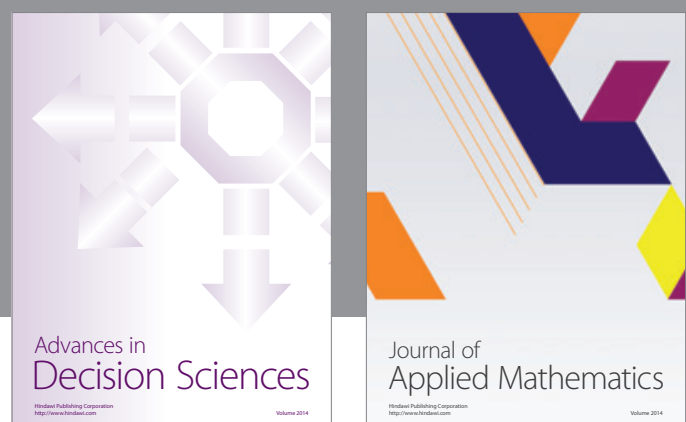

Journal of

Applied Mathematics
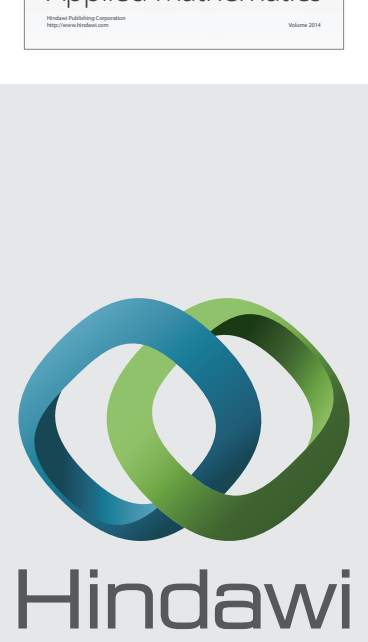

Submit your manuscripts at http://www.hindawi.com
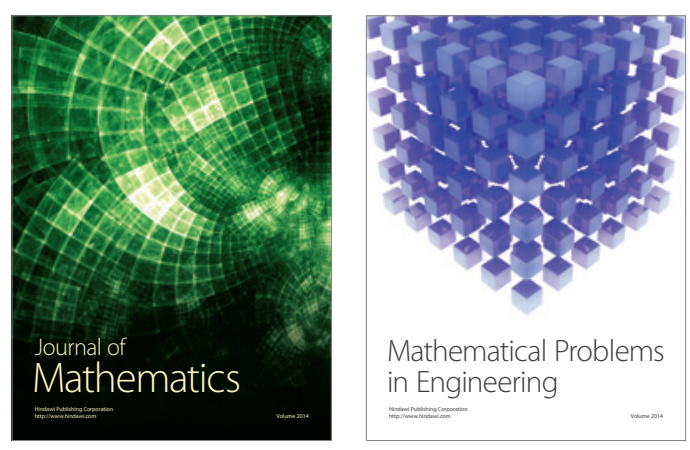

Mathematical Problems in Engineering
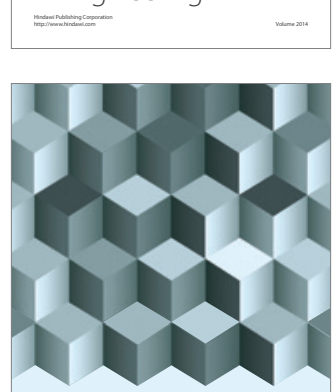

Journal of

Function Spaces
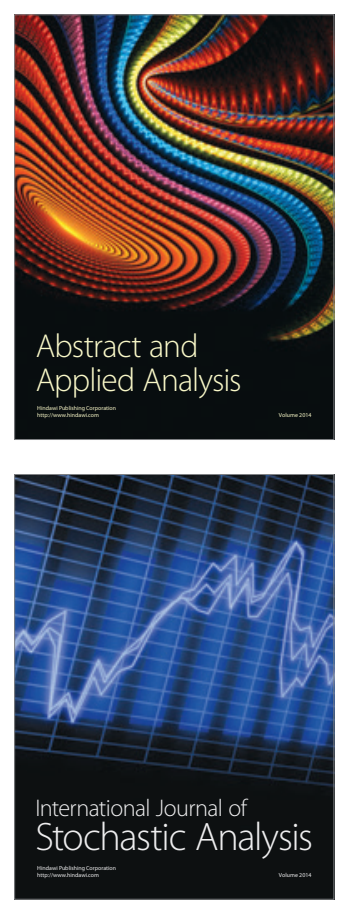

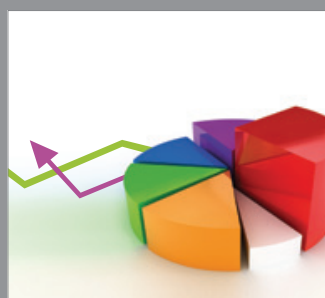

ournal of

Probability and Statistics

Promensencen
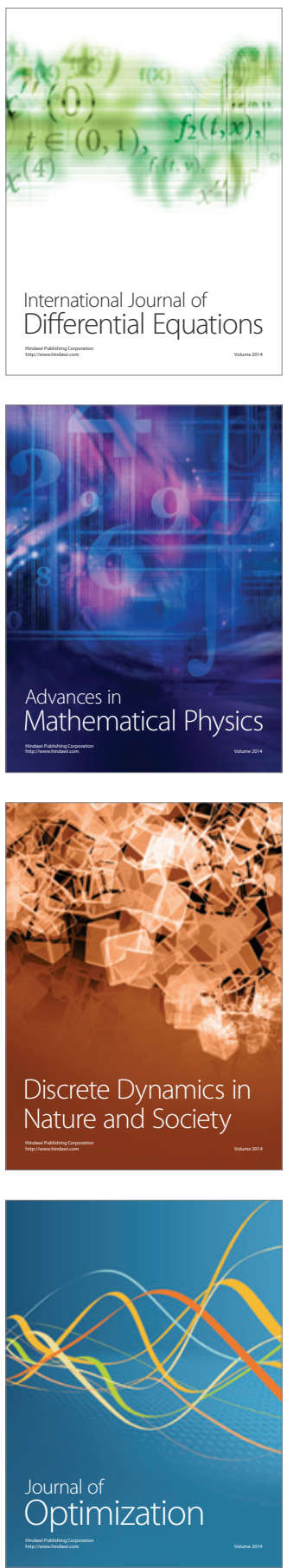